DESY M-89-23

October 1989

\title{
Status and Future of the 3D MAFIA Group of Codes
}

M. Dehler, M. Dohlus, A. Fischerauer, G. Fischerauer, P. Hahne, R. Klatt, F. Krawczyk, T. Pröpper, P. Schütt, T. Weiland

Technische Hochschule Darmstadt

F. Ebeling, M. Marx, S.G. Wipf

Deutsches Elektronen-Synchrotron DESY, Hamburg

B. Steffen

Kernforschungsanlage Jülich

T. Barts, J. Browman, R.K. Copper, G. Rodenz, D. Rusthoi

Los Alamos National Laboratory, New Mexico, USA 
DESY M-89-23

Oktober 1989

\title{
Status and Future of the 3D MAFIA Group of Codes
}

M. Dehler, M. Dohlus, A. Fischerauer, G. Fischerauer, P.Hahne, R. Klatt, F. Krawczyk, T. Pröpper, $P$. Schütt, T. Weiland

Technische Hochschule Darmstadt, Fachbereich 18,

Fachgebiet Theorie Elektromagnetische Felder, Schloßgartenstr. 8, D-6100 Darmstadt, Germany

\author{
F. Ebeling, M. Marx, S. G. Wipf \\ Deutsches Elektronen-Synchrotron DESY \\ Notkestr. 85, D-2000 Hamburg 52, Germany \\ B. Steffen \\ Kernforschungsanlage Jülich KFA, \\ D-5170 Jülich, Germany
}

T.Barts, J.Browman, R.K.Cooper, G.Rodenz, D.Rusthoi

Los Alamos National Laboratory

Los Alamos, New Mexico 87544, USA

Talk presented at the COMPUMAG Conference on the Computation of Electromagnetic

Fields, Tokyo, Japan, Sept. 3-7, 1989 


\section{STATUS AND FUTURE OF THE 3D MAFIA GROUP OF CODES}

M.Dehlcr, M.Dohlus, A.Fischerauer, G.Fischcraucr, P.Hahne, R.Klatt, F.Krawczyk, T.Pröppcr, P.Schïtt, T.Wciland Technische Hochschnle Darmstaclt, Fachiocreich 18, Fachgebiet Theorie Electromagnetische Felder, Schloßgartcnstr. 8, D-6100 Darmstadt, Germany

F.Ebeling, M.Marx, S.G.Wipf

Deutsches Elektronen-Synchrotron DESY, Notkestr. 85, D-2000 Hamburg 52, Germany

B.Stcffen

Kernforschungsanlage Jülich KFA, D-5170 Jülich, Germany

T.Barts, J.Browman, R.K.Cooper, G.Rodenz, D.Rusthoi

Los Alamos National Laboratory, Los Alamos, New Mexico 87544, U.S.A.

\section{ABSTRACT}

The MAFlA group of fully three-dimensional computer codes for solving Maxwell's equations is well established. Extensive comparisons with measurements have demonstrated the accuracy of the computations. The latest additions include a static solver that calculates 3-D magneto- and electrostatic fields and a self-consistent version of TBCII that solves the field equations and the equations of motion in parallel. Work on new eddy-current modules has started, which will allow treatment of laminated and/or solid iron cores excited by low-frequency currents. Based on our experience with the present releases 1 and 2, we have begun a complete revision of the whole user interface and data structure.

\section{Introduction}

The acronym MAFIA stands for the solution of MAxwell's equations by the Finite Integration Algorithm. This is the name given to a set of fully three-dimensional codes widely used in the computeraided design of accelerators and rapidly finding application in many other areas such as tomography and the design of integrated circuits, filters and resonators.

\section{The Method}

The MAFIA programs solve Maxwell's equations directly, rather than using such equations as the Poisson or Wave equations, which are derived from the Maxwell equations under certain conditions. This means that the solutions obtained can be applied to the whole geometrical range modelled by the code, and that transitions between materials need not be treated separately.

The allocation of the field components to the rectangular grid G, uses the Yee Lattice [1] as shown in Figure 1, with the electric field components allocated at the mid-points of the sides of the rectangular cells and the magnetic field components at the centre of each face, defining a dual grid, $\tilde{G}$.

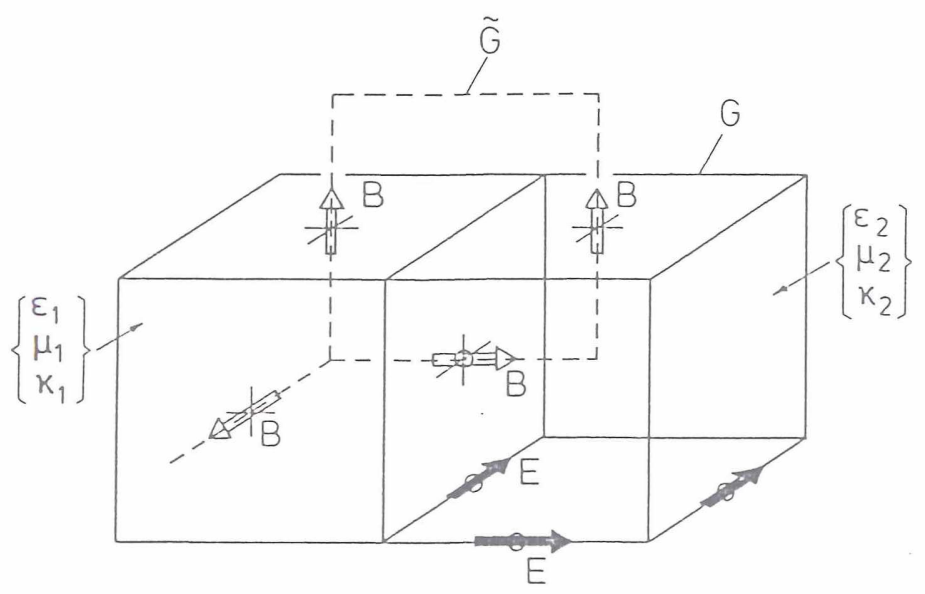

Figure 1: Geometry and Allocation of the Field Components for the FIT [3] Methorl
The advantage of this allocation is that, wherever there is a transition from one material to another, only electric field components parallel to the material surface or magnetic filed components perpendicular to the surface occur. Thus Maxwell's equations are always satisfied at the points of allocation, even when different materials are involved.

The Finite Integration Technique (FIT) $[2,3]$ produces a first order approximation of Maxwell's equations by replacing the line and surface integrals (1)-(4) in Real Space by mean field values multiplied by path lengths or areas in Grid Space.

\section{The Grid Equations}

$$
\begin{aligned}
& \text { Real Space } \\
& \int_{(A)} \mathrm{E} \cdot d \mathrm{~s}=-\iint_{A} \frac{\partial \mathrm{B}}{\partial t} \cdot d \mathrm{~A}=C \mathrm{D}_{s} \mathrm{e}=-\mathrm{D}_{\mathrm{A}} \dot{\mathrm{b}} \\
& \oint_{(A)} \mathrm{H} \cdot d \mathrm{~s}=\iint_{A}\left(\frac{\partial \mathrm{D}}{\partial t}+\mathrm{J}\right) \cdot d \mathrm{~A}=\tilde{\mathrm{C}} \tilde{\mathrm{D}}_{\mathrm{s}} \mathrm{h}=\tilde{\mathrm{D}}_{\mathrm{A}}(\dot{\mathrm{d}}+\mathrm{j}) \\
& \iint_{(V)} \mathrm{B} \cdot d \mathrm{~A}=0=\mathrm{SD}_{\mathrm{A}} \mathrm{b}=0 \\
& \iint_{(V)}\left(\frac{\partial \mathrm{D}}{\partial t}+\mathrm{J}\right) \cdot d \mathrm{~A}-0 \quad \tilde{\mathrm{S}} \tilde{\mathrm{D}}_{\mathrm{A}}(\dot{\mathrm{d}}+\mathrm{j})=0 \\
& \mathrm{D}=\mathrm{c} \mathrm{E} \Rightarrow \mathrm{d}=\mathrm{D}, \mathrm{e} \\
& \mathrm{B}=\mu \mathrm{H} \Leftrightarrow \mathrm{b}=\mathrm{D}_{\mu} \mathrm{h} \\
& \mathrm{J}=\kappa \mathrm{E}+\rho \mathrm{v} \Leftrightarrow \mathrm{j}=\mathrm{D}_{\kappa} \mathrm{e}+\mathrm{D}_{\rho \mathrm{V}} \\
& \text { div curl } \equiv 0 \Leftrightarrow S C=\tilde{S} \tilde{C}=0 \\
& \text { curlgrad } \equiv 0 \quad \Leftrightarrow \quad \tilde{C}^{t} \tilde{S}^{t}=C^{t} S^{t}=0
\end{aligned}
$$

For the left hand side eqations; $\mathbb{E}$ and $\boldsymbol{H}$ are the electric and magnetic field strength, $\mathbb{D}$ and $\mathrm{B}$ the flux densities and $\mathrm{J}$ the current density. Field strengths, flux densities and current densities are related through Equations (5)-( 7 ) where $\epsilon, \mu$ and $\kappa$ are the permeability, permit.livity and conductivity of the materials. SI units are used.

For the matrix equations on the right hand side; the mesh stcp sizes for the integration path are put into a diagonal matrix, $D_{s}$ while $D_{A}$ contains the surfaces of the grid cells and the matrix $\mathrm{C}$ replaces the contour integral operator in Equation (1) for all mesh points. All unknown components of the electric field $\mathbb{E}$ for each mesh point are put into a vector $\mathrm{e}$, and we replace $\mathrm{D}$ by $\mathrm{d}, \mathrm{B}$ by $\mathrm{b}, \mathrm{H}$ by $\mathrm{h}$ and $\mathrm{J}$ by $\mathrm{j}$ respectively. The matrices $\tilde{D}_{\mathrm{s}}$ and $\tilde{\mathrm{D}}_{\mathrm{A}}$, which hold the mesh step sizes and cell areas for the dual grid $\tilde{G}$, are defined similarly. A full description of the derivation of these equations can be found in $[4,6]$.

\section{Properties of the Grid Equations}

One of the outstanding properties of Maxwell's grid equations is that the analytical properties of the solutions, in particular equations (8) and (9), are preserved on the discrete grid $[2,4]$. This is not necessarily the case for all discretisation methods.

These propertics of the Maxwell grid equations not only offer a unigue tool to test numerical results for their plyssical correctness but also guarantee the identification of incorrect solutions in the calcula- 
tion of three-dimensional eigenmodes in resonators [4]. In addition, as one often investigates unknown phenomena by using such codes, pinpointing incorsect solutions helps to prevent physical misinterpretations.

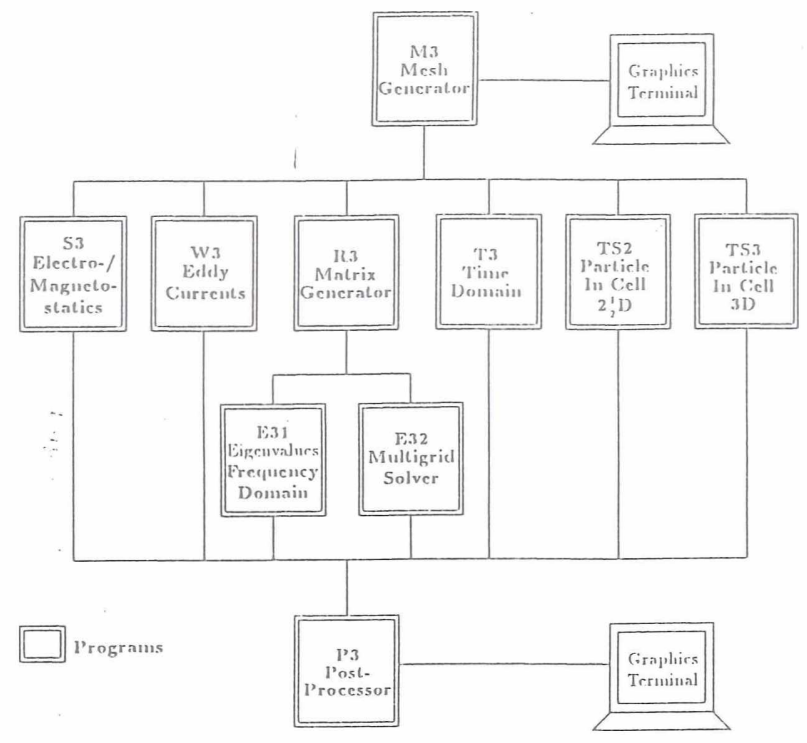

Figure 2: The MAFIA System with its Inter-relalionships

\section{Description of the MAFIA Programs}

- M3 is the mesh generator used by all programs, which translates the physical problem into mesh data and material distribution data. In the new release the user will have the possibility of entering the geometry of the problem interactively with the help of the internal editor and the diagnostic checks built into the program. A command file will then be crealed for subsequent. use.

- S3 solves three-dimensional electro- and magneto-static problems. This program also allows the calculation of stationary temperature and current. distributions $[9,10]$.

- W3 will solve Maxwell's equations for eddy currents |7], (not yet available for public release).

- R3 and E31 (or E32), these two codes, run in sequence, solve Maxwell's equations in the frequency domain. R.3 sets up the matrices, adding the material properties and the boundary conditions. E31 is the more accurate eigenvalue solver, which solves the matrix equations and writes the required eigenvectors onto the direct access file. E32 uses advanced multigrid methods and may be used instead of E31 for very large meshes.

The new release allows lossy materials (complex $c$ and $\mu$ and non-zero conductivity). This is achieved by using truly complex field vectors rather than by introducing losses via a perturbation method. Furthermore, two dimensional applicalions such as waveguide modes or cylindrical geometries are being included, covering the capabilities of the stand-alone $2 \mathrm{D}$ codes URMEL [11] and URMEL-T [12].

- T3 solves Maxwell's equations in the time domain [5,8]

- TS2 is a two and a half dimensional particle-in-cell code that solves Maxwell's equations in the time domain including both particle fields and motion. This code can simulate, for example, radio frequency tubes and particle sources in a fully selfconsistent way.

- TS3 is a three-dimensional particle-in-cell code, (not yet available for public release).

- P3 is the postprocessor for all the codes. Solutions can be displayed graphically and calculalions carried out.
Figures 3 to $\bar{i}$ show trpical out put from the programs from calculations which have heen carried out for the. IILRA accelerating ring at DEST. Figure 3 represents a correction miagree for the dectron ring calculated hy $\$ 3$. While Figure 4 shows the arrow plots of the the magnetic flux in a vertical cross-section. Figure 5 shows output from T3. The structure in Tigure 6 was a proposed kicker magnet for injection of protons from PETRA into the HERA ring, where the nagnet is carried on supports which rotate it into the beam line; however, the computer simulations, using R3-E3, showed that parasitic If impedances were far too high to ensure a stable beam. Thus this version of the magnot was not built and a new one was designed and built using MAFIA. The arrow plots in Figure $\tau$ show the electric and magnetic fields across the gaj, of the magnet.

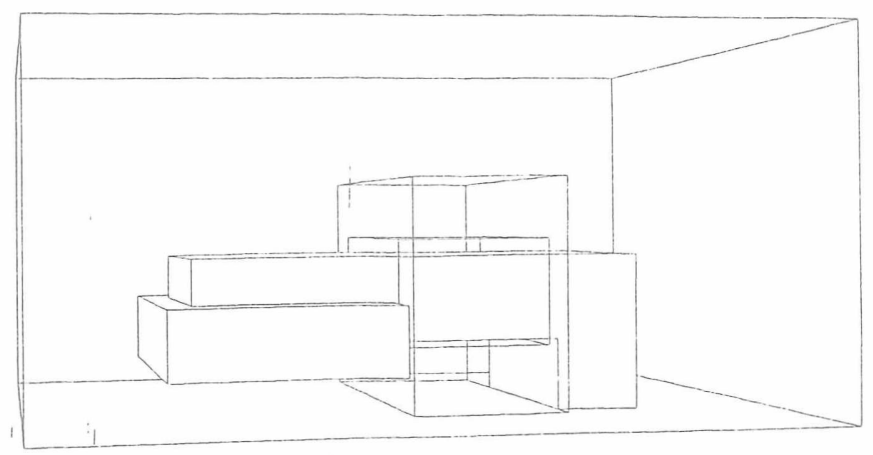

Figure 3: Geometry of a lIERA Correction Dipole Magnet calculated by $\$ 3$

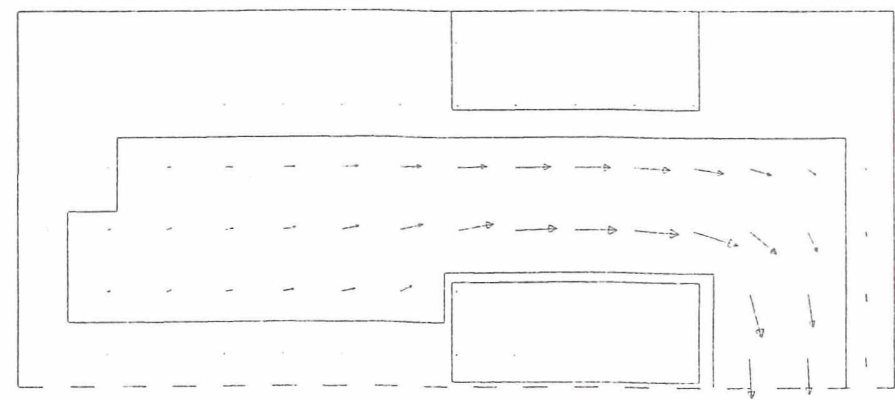

Figure 4: Arrow plots, from P3, showing the Magnetostatic Field calculated by $S 3$, in a vertical plane through the geometry of Fig. 3

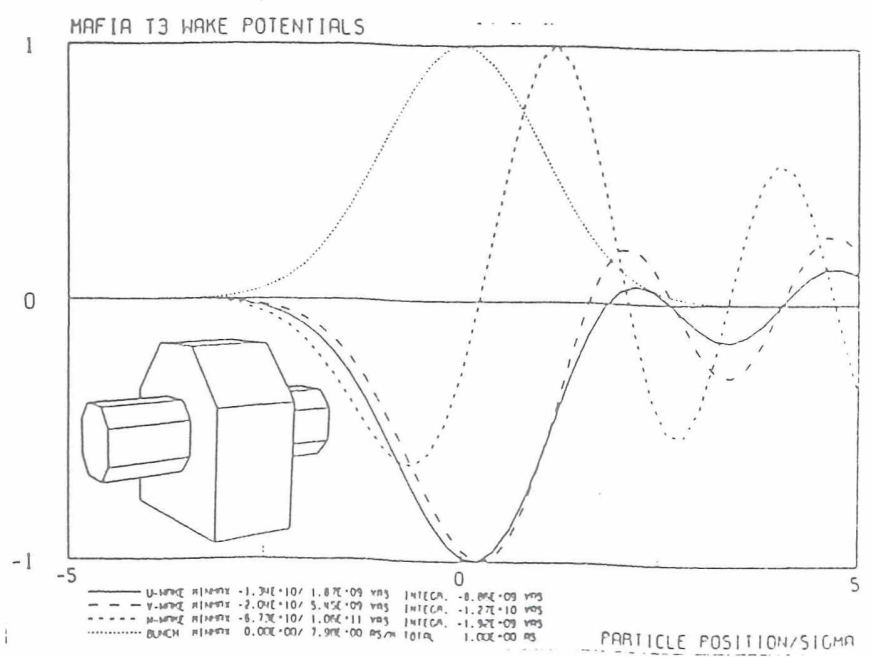

Figure 5: Wakefield Potentials versus bunch coordinates from $\mathrm{T} 3$, for the geometry shown in the insert 


\section{Present Status}

The second release of the MAFIA Programs, (omujrising M3, R.3, E31, E32 and P3. has been distributed to over 320 installations in 18 countries, including NMFE computer Center in the United States. The programs have already proved thejr dependability and usefulness through comparison with theoretical calculations and by successful design of accelerator components. For the third release, the existing MAFIA codes are being completely overhauled and new codes added. However the internal physics routines have been left unchanged, so the dependability of the results is not at risk.

\section{Features of the New Release of the Programs}

- A modular program structure has been adopted.

- A unified. menu-controlled. user interface has been created for all the codes.

- A new command processor has been writtcn.

- The data structure has been changed to accomodate all codes.

- A dynamic memory manager has been added to control both the file and the memory operations.

- Two dimensional geometries will also he included.

\section{User Interface}

In order to create a system with a clear, consistent logical structure which will enahle casy extension of existing programs and áddition of new ones, the programs have been restructured and divided into stctions. Each of these sections represents an independent function of the program, for example, the setting of the mesh or the drawing of an arrow plot. The new user interface is guided by menus, which list all the commands available in a section, with their current settings or default values. This reduces the memorisation of command names, and displays the status information for the section. All sections have the same basic structure -

menu display
reading and processing of command lines
setting of appropriate variables
optional execution or exit

It is also possible to run the programs without the menu display, in this mode the information about the values set for certain commands can still be obtained on request. A certain subset of commands, which it is desirable to have available at any time, have been selected and called global commands. For example, the menu display may be switched off or the file directory printed using a global command. An escape character defined in the user interface will enable certain functions of the system interface to be invoked from within the program. In addition, a section named Private will be provided as an open software interface to enable users to add their own sections to the program.

\section{Help system}

The user interface includes a hierarchical help system for the heginning user. The most basic help answers such questions as: - What does this program do? - How do I use it? At any stage of the program the command $h c l p$ alone will give advice on how to proceed. A description of the function of each section together with the meaning of the subcommands is also available.

\section{Command Processor}

The command processor was designed wjth two aims in mind: to define command names, wherever possible. whose function shall be obvious and to minimise the necessary typing for intcractive use. All commands are defined with unabbreviated names. This relicves the user of the task of memorising specific command numemonics. The command processor is capable of processing shortened versions of these commands; it checks for validity and ambiguity and will accept any mmannbignous truncation. More than one command may be entered on a line. Thus in many sections a single letter will suflice to define a command and it hecomes possible to include many commands in a single juput line. The functions of the commands are also, as far as possible, independent, so that the order of commands is not predetermined: however complete flexilbility in this respect is not possible - a file must be opened hefore it is read and read before a calculation can be carrjed out. In order to kecp) the programs as streamlined as possible, the idea of interactive prompting for missing information was rejected as impractical and time-consuming. Certain necessary checks will be made when the reguest 1.0 execute a section is received and warning messages issued.

A macro facility will be included and the command processor will be able to accept. predefined macro commands, standing for a string of basic commands. This will enable experienced users to streamline terminal sessions and tailor the program to their particular needs.

\section{Data structure of the direct accoss file}

The new direct access file has a transparent structure with a directory, organised like a database. The storage locations of named fields are recorded in the directory and the user has control over the reading and writing of quantities on the filc. The directory is stored and updated in memory and written to the file when the program is terminated. Much general information stored on the file, such as the number of mesh points and the boundary conditions, are stored in readable form so that they can he listed on request from inside the program. However, many systems do not support expandahle direct access files. While it would be possible to work with one file which is large enough for all the programs. it is unwicldy to do so and often it is not obvious, when M3 is started, how large the final file should be. For this reason, it will be possible to allocate, open, close and extend direct access files from within the MAFIA programs.

\section{Sequential Files}

The following sequential files are provided for:

- Command file - Commands may be read from this file instead of from the terminal. This will replace the former input files for M3, R3 el.c. A command file could be used several times to make a set of arrow and contour plots for the same geometrical values, or more than one command file may be used in a session to perform various tasks.

- Profile - When this file is present, it will be read and processed before interaclive control begins. This is basically another command file which has been found nccessary, due to the cnormous variation at different installations in filc handling and interactive capabilities. For cxample, personal macros can be defined here, global commands set or terminal types defined. Thus the programs can be set up to behave as the local users would wish and expect.

- Log file - When this file is open, all the commands entered will be written onto it. This is also a useful method of creating a command file for future usc as well as of recording the progress of a session. The commands used during a session are also automatically recorded on the direct access file when the program is terminated. This is to aid in the diagnostic work of following up on user-reported problems which are often not otherwise exactly reproducible.

- Print file - Interactive output may be recorded on a print file and the interactive termimal may be switched off by a global command when output is produced for later study.

- Plot file - Graphics is handled by means of a system of workstations. The user may select interactively any type of workstation for graphics out.put such as a screen or a file.

There is also an internal editor avajlable where the contents of any text files can be listed, changed if necessary, rewritten for later use. In this manner the contents of a command file could be checked or 
altered before it is used or a logfile could be reused as a command file to repeat a complicated series of commands.

\section{Dynamic Memory Manager}

Ore of the restrictions of three dimensional mesh codes in general is the number of mesh points which can be handled on any particular system. The more mesh points available. the greater the accuracy obtainable in the approximation of the structure. One of the aims of the new file structure and memory manager is 10 provide the maximum flexibility in the use of available memory. The dynamic memory manager allots space in core for each operation and any temporarily used space can be immediately released for reuse. A combination of selective loading of quantities from the file and of selective deletion in memory of whatever is not needed for a particular opcration, can make optimum use of the space available. A load section is provided, where.the normal file and memory operations, e.g. get, replace, delete, compress et.c., can be invoked.

\section{Mathematical Operations}

Many users found that further calculations were necessary to adapt the quantities calculated by $P 3$ to their needs. In the future such quantities will be programmed at the level of the individual mathematical operations. This facility will also be made available to the user. It will be possible, for example, to load a field from one direct access file, load the corresponding ficld from a file with the same mesh but slight.Jy different geometry, difference the two and display the result graphically or perform further calculations as needed.

\section{Availability}

The MAFIA codes are written in standard FORTRAN $7 \tau$ and currently run on IBM, CRAY, VAX, APOLLO, HP. SUN, CONVEX, AMDAHL, FUJITSU. IHTAC'HI and STELLAR computers, among others. The distribution centre for the codes is the Technische Hochschule, Darmstadt, for information contact Thomas Weiland there.

\section{Conclusion}

Many new features will be added to the MAFIA group of codes. The additional programs will extend the scope of the codes while the new command processor, the conscious file manipulations and the additional calculations that will be possible, will be the features affecting users most directly. However the underlying restructuring of the programs themselves enables a much more efficient management of the support of the codes and is flexible enough to accomodate virtually any future extensions. The newest release of the MAFIA codes will be more user friendly than before, while the new flexibility will allow users to adapt it to their own particular needs.

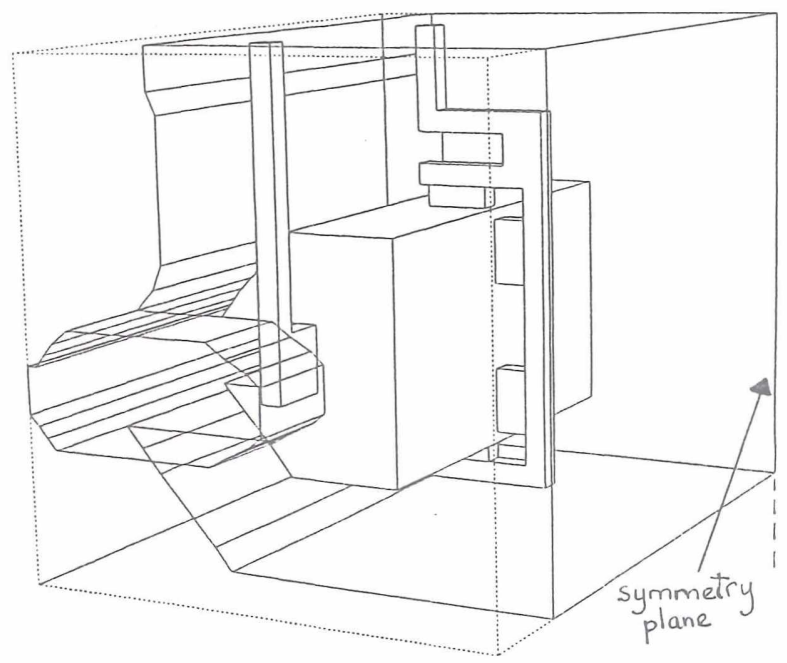

Figure 6: Structure of a Proposed Kïker Magnet for Injection into HERA, (one half of the structure shown)

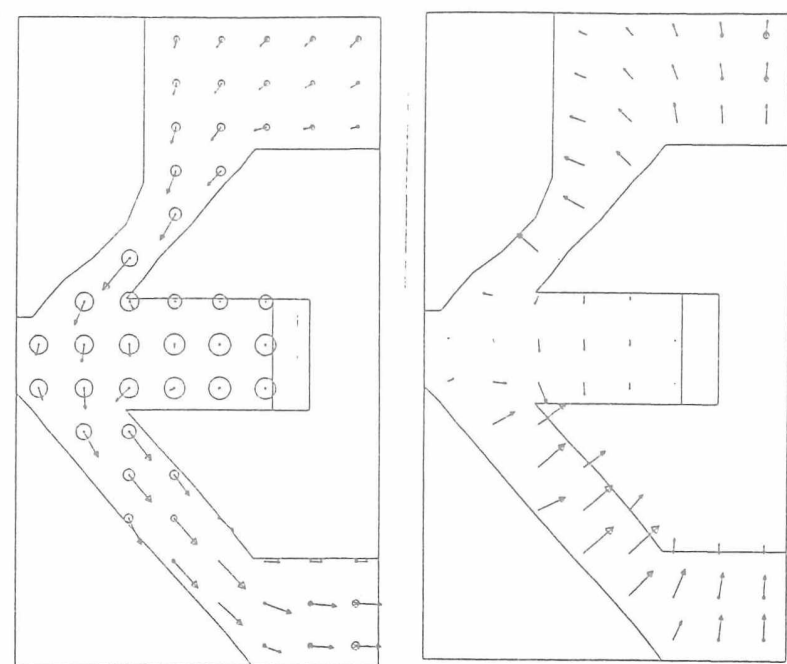

Figure 7: Arrow plots of electric and magnelic field. from E3 - I'3 for one of the parasitic of modes in the kicker magnet(fig.6)

\section{References}

[1] K.S.Yec. "Numerical Solution of Infinite Boundary Value Problems lnvolving Maxwcll's Equations in Isotropic Media," IEEE, AP-14, 1966, p).302-307.

[2] T.Weiland, "On the Numerical Solution of Maxwell's Equations and Applications in the Field of Accelerator Physics," Particle Accelcrators 15 (1984), pp.245-292 and references therein

[3] T. Weiland, "On the Unique Solution of Maxwellian Eigenvalue problems in Three Dimensions." Particle Arrelerators 17 (1985). pp. $22 T-242$

[4] T.Weiland: "Numerical Solution of Maxwell's Equations For Static, Resonant and Transient Fields," Proceedings of the International URSI Symposium on Electromagnetic Theory, pp.53i-542, Budapest, Aug. 1986

[5] T.Weiland: "Transient Electromagnetic Fields Excited by Bunches of Charged Particles in Cavities of Arbitrary Shape," Procecdings of th XI-th International Conference on High Energy Accelerators, Geneva 1980, Birkhauser Verlag. Basel pp 570 .

[6] T.Weiland: "A Discretization Method for the Solution of Maxwell's Equations for 6 Component Fields," $\mathrm{AEÜ,}$, Elect.ron. and Commun., 31(197i),pp.116-120

[i] H.Euler und Th. Weiland: "On the Calculation of Eddy Currents in Arbitrarily Shaped, Three Dimensional, Laminated Iron Cores." Archiv für Elektrotechnik (AfE), Vol.61(1979), pp.103 (see also Vol.65(1982),pp.299)

(8) R.Klatt. T.Weiland, "Electromagnetic design of the Hera eVacumn System." SLAC: Report 303, p.282 (1986)

[9] F.Krawczyk und Th. Weiland: "A New Static Solver with Open Bonndary Conditions in the 3D-CAD-System MAFIA." IEEE, Trans.in Mag.22/1(1988) pp.55-5i

[10] F.Krawczyk und Th. Weiland: "Use of the Multigrid Solver in the MAFIA Module $\$ 3$ for Electro and Magnetostatic Problems," Contribution to the Compumag Conference, Tokyo, Japan Sept.1989, see forthcoming proccedings.

[11] T.Weiland. "On the Compulation of Resonant Modes in Cylindrically Symmetric Cavities," Nuclear Instruments and Mothods NIM 216 (1983), pp.329-348

[12] U.van Rienen. T. Weiland, "Triangular Discretization Method for the Evaluation of RF-Fields in Waveguides and Cylindrically Symmetric Media," Particle Accelerators 20 (1986/1987), pp.239-267

[13] "MAFIA IIser Guide," The Mafia Collaboration, DESY, I Jos Alamos National Lahoratory, KFA-Jülich, February 23, 1987 